\title{
Expression profiling of 22 genes involved in the PI3K-AKT pathway identifies two subgroups of high-grade endometrial carcinomas with different molecular alterations
}

\author{
Lluis Catasus, Emanuela D’Angelo, Cristina Pons, Iñigo Espinosa and Jaime Prat
}

Department of Pathology, Hospital de la Santa Creu i Sant Pau, Autonomous University of Barcelona, Barcelona, Spain

\begin{abstract}
Previously, we showed that PIK3CA and p53 alterations in uterine endometrial carcinomas correlate with poor prognosis. However, the contribution of phosphatidylinositol 3-kinase (PI3K) -AKT deregulation to endometrial carcinogenesis is not completely understood. The purpose of this study was to analyze alterations of this pathway in endometrial carcinomas and correlate them with the most common genetic abnormalities. Expression profiling of 22 genes involved in PI3K-AKT signaling pathway was analyzed in 38 endometrial carcinomas using TaqMan low-density array (TLDA) analysis. The gene expression pattern was analyzed by hierarchical clustering analysis. Unsupervised clustering divided the high-grade endometrial carcinomas into two clusters. One cluster identified tumors with alterations in the PI3K-AKT signaling pathway (exon 20 PIK3CA mutations and/or PTEN mutations $9 / 15 ; 60 \%)$, and p16 protein overexpression $(8 / 13 ; 62 \%)$. Almost all nonendometrioid adenocarcinomas (serous and clear cell adenocarcinomas) were segregated into this cluster. In contrast, the other cluster identified tumors with p53 alterations $(6 / 6 ; 100 \%)$, p16 protein overexpression (5/5; 100\%), and exon 9 PIK3CA mutations (2/6; 33\%). Exon 20 PIK3CA and PTEN mutations were not found in this subgroup. Low-grade endometrial carcinomas clustered in a third subgroup characterized by high frequency of PTEN mutations $(10 / 17 ; 59 \%)$ and microsatellite instability $(6 / 17 ; 35 \%)$. Our results show that gene expression profile differences in the PI3K-AKT signaling pathway identify two subgroups of high-grade endometrial carcinomas with different molecular alterations (PI3K-AKT pathway vs p53 alterations) that may have distinct roles in endometrial carcinogenesis. Identification of these subgroups can provide insight into the biology of these tumors and may facilitate the development of future treatments.
\end{abstract}

Modern Pathology (2010) 23, 694-702; doi:10.1038/modpathol.2010.44; published online 19 February 2010

Keywords: endometrial carcinoma; PI3K-AKT pathway; TLDA; clustering analysis; PIK3CA; p53

The phosphatidylinositol 3-kinase (PI3K)-AKT signaling pathway regulates the expression of several downstream target genes that inhibit apoptosis and promote cell proliferation. ${ }^{1,2}$ The effect of its deregulation on the clinical outcome of human tumors has recently become a subject of intense investigation.

Correspondence: Professor J Prat, MD, PhD, FRCPath, Department of Pathology, Hospital de la Santa Creu i Sant Pau, Avenida Sant Antoni Maria Claret 167, Barcelona 08025, Spain.

E-mail: jprat@santpau.cat

Received 20 October 2009; revised and accepted 27 January 2010; published online 19 February 2010
Activation of this pathway has been associated with aggressive phenotype and poor prognosis in brain tumors (glioblastoma and neuroblastoma) as well as in various carcinomas, including breast, prostate, bladder, colon, and lung carcinomas. ${ }^{3-9}$

Endometrial carcinoma is the most common malignant tumor of the female genital tract and the fourth most common female cancer in the Western World. ${ }^{10}$ It has been divided into two major types. ${ }^{11-13}$ Type I carcinomas are largely low-grade endometrioid carcinomas that occur in pre- and perimenopausal women. They are estrogen dependent, frequently preceded by endometrial hyperplasia, 
and characterized by microsatellite instability, PTEN, PIK3CA, CTNNB1, and K-RAS mutations. ${ }^{14-19}$ Type II carcinomas are high-grade non-endometrioid carcinomas that develop in post-menopausal women and are commonly associated with p53 alterations. ${ }^{20-22}$ Although surgery is still the main form of treatment, some patients with advancedstage tumors have benefited from chemotherapy. ${ }^{23,24}$

Recently, we reported that PIK3CA mutations are frequent events in endometrial carcinomas of any histological type. Whereas carcinomas with exon 20 PIK3CA mutations or PIK3CA mRNA overexpression are often high-grade carcinomas associated with myometrial invasion, tumors that carried exon 9 mutations are more likely low-grade endometrioid carcinomas. ${ }^{16}$ We also found that alterations in the PI3K-AKT signaling pathway (PIK3CA and/or PTEN mutations) alone did not influence overall survival; however, patients with deregulated PI3K-AKT pathway who also had p53 alterations had shorter survival than patients with only p53 alterations. ${ }^{25}$

Nevertheless, the role of deregulations of the PI3K-AKT signaling pathway in the pathogenesis of endometrial carcinoma is only partially understood, as unrecognized alterations in less popular genes may be involved in tumor development. In an attempt to uncover new alterations of the PI3K-AKT pathway, we have examined the expression profiling of 22 involved genes in 38 endometrial carcinomas by Taqman low-density array (TLDA). Subsequently, we analyzed the gene expression pattern by a hierarchical clustering analysis and correlated the results with the clinicopathological features of the tumors and their molecular genetic alterations.

\section{Materials and methods}

\section{Selecting Tissue Samples}

Samples from 38 endometrial carcinomas were retrieved from the Tumor Bank and the Surgical Pathology files of Hospital de la Santa Creu i Sant $\mathrm{Pau}$, Barcelona, Spain. All cases were reviewed and classified using the 2003 World Health Organization (WHO) criteria. Tumors showing crowded glands resembling those of the normal endometrium were diagnosed as endometrioid adenocarcinomas. They were classified as high grade when the glandular differentiation decreased and the glands were replaced by solid nests. The term serous carcinoma was applied to tumors composed of cells with marked nuclear atypia, cellular budding, and psammoma bodies. The diagnosis of clear cell adenocarcinoma was made when the tumor consisted of clear or hobnail cells with large and pleomorphic nuclei and prominent nucleoli. Mixed adenocarcinomas were defined as neoplasms with endometrioid and non-endometrioid components with the minor component comprising at least $10 \%$ of the total volume of the tumor. We selected a similar number of low-grade and high-grade carcinomas. High-grade tumors $(n=21)$ consisted of 8 grade 3 endometrioid adenocarcinomas, 8 non-endometrioid adenocarcinomas (4 serous, 3 clear cell, and 1 mixed serousclear cell adenocarcinoma), and 5 mixed endometrioid and non-endometrioid adenocarcinomas. All low-grade tumors were grade 1 and 2 endometrioid adenocarcinomas $(n=17)$. Cases were anonymized and the study was approved by the institutional ethics committee. The cases had previously been subjected to determination of microsatellite instability status, and analysis of mutations in the PTEN, PIK3CA, and p53 genes. ${ }^{16,25}$

\section{RNA Extraction and cDNA Synthesis}

Total RNA from tumors and corresponding nontumor tissues was extracted from fresh frozen biopsies using Trizol reagent (Invitrogen, Carlsbad, CA, USA) and the RNeasy mini kit (Qiagen, Hilden, Germany) as specified by the manufacturer's instructions. RNA was eluded in $25 \mu \mathrm{l}$ of RNase-free water. RNA yield and quality were assessed by agarose electrophoresis and spectrophotometry, and then stored at $-80^{\circ} \mathrm{C}$. RNA was digested with DNase I (Invitrogen). Total RNA of $1 \mu \mathrm{g}$ was used for cDNA synthesis according to the protocol provided with the High Capacity cDNA Reverse Transcription kit (Applied Biosystems, Foster City, CA, USA). Recombinant RNasin ribonuclease inhibitor (Applied Biosystems) was added to prevent RNase-mediated degradation. The cDNA was stored at $-20^{\circ} \mathrm{C}$.

\section{Gene Expression Analyses}

Gene expression analyses were performed at mRNA level by TLDA. Pre-designed TaqMan probe and primer sets for target genes were chosen from an on-line catalog (Applied Biosystems). Once selected, the sets were factory loaded into the 384 wells of TLDA cards. Each TLDA card was configured into eight identical 24 gene sets in duplicate. In all, 22 genes were chosen based on literature reviews of the PIK3-AKT, apoptosis, and cell cycle molecular pathways and their involvement in carcinogenesis. $^{1,2,26,27}$ Each set of genes also contained two housekeeping genes, GAPDH and $A B L-1$ (Table 1). We mixed $5 \mu \mathrm{l}$ of single-stranded cDNA (equivalent to $100 \mathrm{ng}$ of total RNA) with $45 \mu \mathrm{l}$ of nuclease-free water and $50 \mu \mathrm{l}$ of TaqMan Universal PCR Master Mix. After gentle mixing and centrifugation, $100 \mu \mathrm{l}$ of mixture was transferred into a loading port on a TLDA card. The card was centrifuged twice for $1 \mathrm{~min}$ at 1100 r.p.m. to distribute the samples from the loading port into each well. It was then sealed and placed in the micro fluidic card sample block of an Applied Biosystems 7900HT PCR system (Applied Biosystems). The thermal cycling conditions were $2 \mathrm{~min}$ at $50^{\circ} \mathrm{C}$ and $10 \mathrm{~min}$ at $95^{\circ} \mathrm{C}$, followed by 40 cycles of $15 \mathrm{~s}$ at $95^{\circ} \mathrm{C}$ and $1 \mathrm{~min}$ at $60^{\circ} \mathrm{C}$. Expression levels were measured in duplicate. Only 
Table 1 Gene expression assays used for configuring the TLDA card

\begin{tabular}{|c|c|c|c|c|}
\hline Gene symbol & Gene name & Ref. sequence & Assay ID & Amplicon length \\
\hline XIAP (BIRC4) & $\mathrm{X}$-linked inhibitor of apoptosis & NM_001167.2 & Hs00236913_m1 & 116 \\
\hline AKT1 & v-akt murine thymoma viral oncogene homolog 1 & NM_005163 & Hs00178289_m1 & 66 \\
\hline TWIST1 & Twist homolog 1 (Drosophila) & NM_000474.3 & Hs00361186_m1 & 115 \\
\hline$B A D$ & BCL2-associated agonist of cell death & NM_004322.2 & Hs00188930_m1 & 69 \\
\hline$C D K N 1 A(p 21)$ & Cyclin-dependent kinase inhibitor 1A (p21, Cip1)) & NM_000389.2 & Hs00355782_m1 & 66 \\
\hline$A B L 1$ & v-abl Abelson murine leukemia viral oncogene homolog 1 & NM_005157.3 & Hs00245443_m1 & $\begin{array}{c}\text { Endogenous } \\
\text { control }\end{array}$ \\
\hline CDH1 & Cadherin 1, type 1, E-cadherin & NM_004360.3 & Hs00170423_m1 & 117 \\
\hline TP53 & Tumor protein p53 & NM_000546 & Hs00153340_m1 & 81 \\
\hline CASP3 & Caspase 3, apoptosis-related cysteine peptidase & NM_004346.2 & Hs00263337_m1 & 111 \\
\hline$P A K 1$ & p21/Cdc42/Rac1-activated kinase 1 & NM_002576.4 & Hs00176815_m1 & 93 \\
\hline$G A P D H$ & Glyceraldehyde-3-phosphate dehydrogenase & NM_002046.3 & Hs99999905_m1 & $\begin{array}{l}\text { Endogenous } \\
\text { control }\end{array}$ \\
\hline PIK3CA & Phosphoinositide-3-kinase, catalytic, $\alpha$-polypeptide & NM_006218.2 & Hs00180679_m1 & 104 \\
\hline FAS & TNF receptor superfamily, member 6 & NM_000043.3 & Hs00531110_m1 & 97 \\
\hline AKT2 & v-akt murine thymoma viral oncogene homolog 2 & NM_001626.3 & Hs00609846_m1 & 129 \\
\hline FRAP1 (mTOR) & FK506 binding protein 12-rapamycin associated protein 1 & NM_004958.3 & Hs00234522_m1 & 71 \\
\hline FOXO1A & Forkhead box $\mathrm{O} 1$ & NM_002015.3 & Hs00231106_m1 & 103 \\
\hline PTK2 (FAK) & PTK2 protein tyrosine kinase 2 & NM_005607.3 & Hs00178587_m1 & 68 \\
\hline CASP9 & Caspase 9, apoptosis-related cysteine peptidase & NM_001229.2 & Hs00154260_m1 & 71 \\
\hline PTEN & Phosphatase and tensin homolog & NM_000314.4 & Hs00829813_s1 & 154 \\
\hline$C C N D 1$ & Cyclin D1 & NM_053056.2 & Hs00277039_m1 & 94 \\
\hline NFKB1 & Nuclear factor $\kappa$-light polypeptide gene enhancer B-cells 1 & NM_003998.2 & Hs00765730_m1 & 66 \\
\hline$G S K 3 B$ & Glycogen synthase kinase $3-\beta$ & NM_002093.2 & Hs00275656_m1 & 73 \\
\hline MDM2 & Mdm2 p53 binding protein homolog (mouse) & NM_002392.2 & Hs01066938_m1 & 150 \\
\hline$C D K N 1 B(p 27)$ & Cyclin-dependent kinase inhibitor 1B (p27, Kip1) & NM_004064.3 & Hs00153277_m1 & 71 \\
\hline
\end{tabular}

TLDA, TaqMan low-density array.

the genes with reproducible amplification curves of both duplicates were analyzed and presented. TLDA cards were analyzed with RQ Manager Software for automated data analysis. Gene expression values RQ were calculated based on the $\Delta \Delta C_{\mathrm{t}}$ method. Delta cycle threshold $\left(C_{\mathrm{t}}\right)$ values, defined as the point at which the fluorescence rises above the background fluorescence, were calculated with SDS 2.3 software (Applied Biosystems). Normal endometrium pool tissue was used as a calibrator and $A B L-1$ housekeeping gene was the reference for normalization. Gene upregulated was arbitrarily designated as $\times 2$ or higher, and gene downregulated as $\leq 0.5$ when compared with the expression of the reference sample.

\section{Hierarchical Clustering Analysis}

Hierarchical clustering analysis of TLDA data was performed using the Cluster program and the results were visualized using the TreeView program from Stanford as previously reported..$^{28}$ Both programs can be downloaded at: http://rana.lbl.gov/eisen/. Clustered data were shown with tumors on the horizontal axis and genes on the vertical axis.

\section{Immunohistochemical Analysis}

Protein p16(INK4a) expression was evaluated by immunohistochemistry on tissue arrays constructed with a tissue arrayer device (Beecher Instruments, Sun Prairie, WI, USA). Immunohistochemistry was performed on paraffin-embedded tissue sections using the EnVision system (Dako, Glostrup, Denmark) and diaminobenzidine as the chromogen. Sections were stained with monoclonal anti-p16 antibody (MTM, Heidelberg, Germany) using optimized protocols. The reactions were carried out in an autostainer immunostainer. The staining intensity and area extent of the immunohistochemistry was evaluated using the semiquantitative scoring system. The stained sections were semiquantitatively scored by two pathologists. The score (H-score) was calculated by multiplying the staining intensity $(0=$ no staining; $1=$ weak; $2=$ moderate; and $3=$ strong) by the percentage of immunoreactive tumor cells (0-100). P16 immunostaining was considered positive when the H-score was $\geq 150$. This cutoff was chosen because it was close to the median protein expression in our cases.

\section{Statistical Analysis}

Statistical analysis was performed using the statistical package SPSS/win 17.0 (SPSS, Chicago, IL, USA). The following clinicopathological parameters were evaluated: age, tumor size, histological type and grade, depth of myometrial invasion, lymphovascular invasion, clinicopathological stage, hormone receptor status, MI status, p16 protein expression, PIK3CA, PTEN, and p53 mutations, and patient outcome. A value of $P \leq 0.05$ was considered statistically significant. 


\section{Results}

\section{Clinical and Pathological Findings}

The clinicopathological features of the 38 endometrial carcinomas are summarized in Table 2. In brief, age of the patients ranged from 35 to 82 years (mean 68 years). Tumor size varied from 1.2 to $11 \mathrm{~cm}$ (mean $5 \mathrm{~cm})$. Of the 38 cases, $25(66 \%)$ were endometrioid, $8(21 \%)$ non-endometrioid (4 serous, 3 clear cell, and 1 mixed clear cell-serous carcinoma), and 5 $(13 \%)$ mixed endometrioid and non-endometrioid adenocarcinomas (3 endometrioid-clear cell and 2 endometrioid-serous carcinomas). Over half $(55 \%)$ of the tumors were grade 3 . Most tumors were FIGO stage I $(n=28 ; 74 \%)$. Myometrial invasion was found in 34 cases $(<1 / 2$ of the myometrial thickness in 17 cases and $>1 / 2$ in 17). Vascular invasion was observed in one third of cases.

\section{TLDA and Clustering Analysis}

Because the expression values of GAPDH showed distinct variations among the samples, $A B L-1$ was exclusively used as the housekeeping gene. In addition, three of 22 genes implicated in the PI3KAKT pathway were excluded because their signal intensities were too weak.

According to the clustering index, samples clustered into three major groups (clusters 1, 2, and 3;

Table 2 Clinicopathological features of 38 endometrial carcinomas

\begin{tabular}{lc}
\hline Age (mean) & 68 years $(35-82$ years) \\
Tumor size (mean) & $5 \mathrm{~cm}(1.2-11 \mathrm{~cm})$ \\
Histological type & \\
EEC low grade (grades 1-2) & $17(45 \%)$ \\
EEC high grade (grade 3) & $8(21 \%)$ \\
NEEC & $8(21 \%)$ \\
Mixed EEC-NEEC & $5(13 \%)$ \\
& \\
Grade & \\
1 & $8(21 \%)$ \\
2 & $9(24 \%)$ \\
3 & $21(55 \%)$ \\
Stage & \\
I & $28(74 \%)$ \\
II & $2(5 \%)$ \\
III & $5(13 \%)$ \\
IV & $3(8 \%)$ \\
Myometrial invasion & \\
No & \\
$<50 \%$ & $4(10 \%)$ \\
$>50 \%$ & $17(45 \%)$ \\
Vascular invasion & $17(45 \%)$ \\
No & \\
Yes & $25(66 \%)$ \\
\hline
\end{tabular}

EEC, endometrioid endometrial carcinoma; NEEC, non-endometrioid endometrial carcinoma.
Figure 1). Cluster 1 consisted of 15 tumors, which were mostly high-grade endometrial carcinomas (14/15; 93\%). They included 1 low-grade endometrioid carcinoma, 5 high-grade endometrioid carcinomas, 7 non-endometrioid carcinomas (4 serous and 3 clear cell carcinomas), and 2 mixed carcinomas ( 1 endometrioid-clear cell and 1 endometrioidserous carcinomas). Except Foxo1A and p27, most genes were highly expressed, particularly $C C N D 1$ and $E-C A D H$, which were consistently upregulated by more than eightfold compared with normal endometrium pool. Cluster 2 contained 17 tumors, which were mostly low-grade endometrioid carcinomas (15/17; 88\%). They included 15 low-grade endometrioid carcinomas, 1 high-grade endometrioid carcinoma, and 1 mixed endometrioid-clear cell carcinoma. These tumors showed upregulation of p21, FAK, PAK1, and CCND1 and downregulation of CASP3, Foxo1A, PIK3CA, p27, and NFKB. Last, cluster 3 contained six tumors, which were mostly high-grade endometrial carcinomas $(5 / 6 ; 83 \%)$. They included 1 low-grade endometrioid carcinoma, 2 high-grade endometrioid carcinomas, 1 nonendometrioid carcinoma (mixed serous-clear cell carcinoma), and 2 mixed carcinomas (1 endometrioid-clear cell and 1 endometrioid-serous carcinomas). These tumors showed overexpression of PTEN and underexpression of XIAP. Median RQ values of analyzed genes according to the three clusters are shown in Table 3 . Plot box graphs of RQ values expression for FOXO1A, FAK, GSK3- $\beta$, and PTEN in the three clusters are shown in Figure 2.

\section{Association of Clusters Subgroups with the Clinicopathological Parameters and Molecular Alterations}

A relationship between the three clustering subgroups, the clinicopathological prognostic parameters, and the most common molecular genetic alterations was analyzed (Figure 1 and Table 4). Histological type and grade varied significantly according to the tumor distribution into the three clusters $(P=0.000$ for both). Most low-grade endometrioid carcinomas $(15 / 17 ; 88 \%)$ were in cluster 2 , whereas the majority of non-endometrioid carcinomas $(7 / 8 ; 88 \%)$ were in cluster 1 . The nonendometrioid carcinomas segregated into this cluster represented a heterogeneous group of tumors composed of four serous, and three clear cell carcinomas. In addition, high-grade endometrioid carcinomas were mainly distributed in cluster 1 $(5 / 8 ; 63 \%)$, whereas mixed tumors were allocated into the three clusters. All grade 1 tumors (8/8; $100 \%)$, and almost all grade 2 tumors $(7 / 9 ; 78 \%)$ were segregated into cluster 2 , whereas grade 3 carcinomas were mostly found in clusters 1 and 3 $(19 / 21 ; 90 \%)$. Vascular invasion was more commonly encountered in cluster 3 tumors $(3 / 6 ; 50 \%)$ than in clusters $1(5 / 15 ; 33 \%)$ and $2(5 / 17 ; 29 \%)$. 


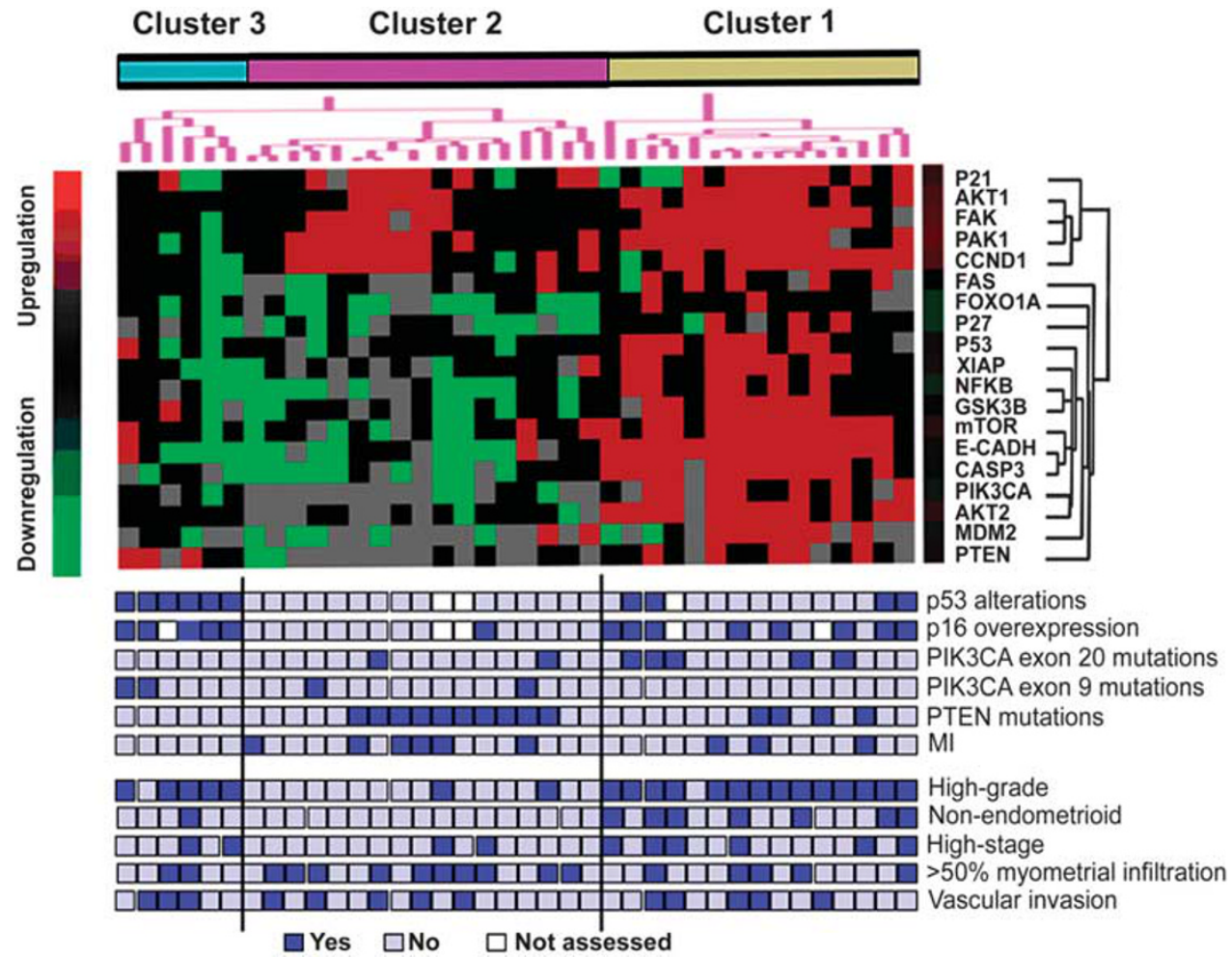

Figure 1 Hierarchical clustering analysis of mRNA expression of 19 genes in 38 endometrial carcinomas. Upregulated and downregulated expression is indicated as red and green cubes, respectively. Genes that did not vary in their expression level are shown in black and genes with unsatisfactory results are labeled in gray. Enclosed in the clustering image, results of p53, p16, PIK3CA, PTEN, and MI analysis as well as clinicopathological parameters, such as high-grade (grade 3), non-endometrioid, high-stage (stage 2 or higher), myometrial invasion ( $>50 \%$ ), and vascular invasion, are graphically represented for each case.

Table 3 Median RQ values of investigated genes (range of values in parentheses) according to the three clusters

\begin{tabular}{lccc}
\hline & Cluster 1 & Cluster 2 & Cluster 3 $^{\text {P-value }}$ \\
\hline CASP3 & $6.58(1.18-27.15)$ & $0.49(0.02-1.33)$ & $0.52(0.33-0.66)$ \\
CCND1 & $9.69(0.42-65.98)$ & $2.78(0.42-7.45)$ & $0.95(0.30-1.43)$ \\
AKT2 & $7.74(1.48-34.51)$ & $0.84(0.13-1.44)$ & $1.22(0.75-1.62)$ \\
XIAP & $2.96(0.99-6.42)$ & $0.90(0.14-2.0)$ & $0.45(0.19-0.78)$ \\
FAS & $3.75(0.28-17.78)$ & $1.58(0.08-9.61)$ & $0.73(0.22-1.28)$ \\
AKT1 & $3.69(1.43-7.66)$ & $1.97(0.54-5.65)$ & $0.79(0.51-1.31)$ \\
MTOR & $5.19(1.43-16.30)$ & $0.91(0.25-3.17)$ & $1.25(0.34-2.74)$ \\
MDM2 & $3.73(0.35-8.12)$ & $1.28(0.22-4.63)$ & $0.92(0.0-1.48)$ \\
GSK3- $\beta$ & $3.67(1.34-12.70)$ & $0.64(0.10-1.64)$ & $1.00(0.09-2.12)$ \\
PAK1 & $2.93(1.10-7.48)$ & $3.18(0.65-10.64)$ & $0.59(0.23-1.08)$ \\
FOXO1A & $1.71(0.52-7.01)$ & $0.43(0.06-1.50)$ & $0.75(0.41-1.33)$ \\
PTEN & $2.76(0.65-10.70)$ & $0.82(0.11-1.76)$ & $2.01(0.73-2.66)$ \\
FAK & $4.24(1.05-8.08)$ & $2.30(1.25-7.46)$ & $0.90(0.37-1.66)$ \\
PIK3CA & $3.15(0.50-7.84)$ & $0.46(0.19-0.63)$ & $0.87(0.37-1.71)$ \\
E-CADH & $15.10(0.50-91.54)$ & $0.69(0.10-2.42)$ & $0.73(0.01-2.56)$ \\
P27 & $1.39(0.38-4.61)$ & $3.89(0.17-1.08)$ & $0.63(0.47-1.02)$ \\
P21 & $3.87(0.30-13.35)$ & $0.85(0.36-16.51)$ & $0.94(0.27-2.08)$ \\
P53 & $3.72(0.72-14.39)$ & $0.39(0.10-0.98)$ & $1.33(0.10-5.42)$ \\
NFKB & $2.12(0.91-6.80)$ & & $0.57(0.31-1.09)$ \\
\hline
\end{tabular}

${ }^{\mathrm{a}} P$-values were calculated using Kruskal-Wallis test.

Upregulated genes (median $R Q \geq 2$ ) are shown in red, downregulated genes (median $R Q \leq 0.5$ ) in green, and genes that did not vary in their expression level (median RQ interval 0.5-2) are shown in black.

Moreover, myometrial invasion ( $>50 \%$ ) was more frequent in cluster 2 tumors $(10 / 17 ; 59 \%)$ than in clusters $1(5 / 15 ; 33 \%)$ and $3(2 / 6 ; 33 \%)$. In contrast, cluster 2 tumors were more frequently low stage (stage $1,15 / 17 ; 88 \%$ vs stage $\geq 2,2 / 17 ; 12 \%$ ) than clusters 1 and 3 (stage $1,13 / 21 ; 62 \%$ vs stage $\geq 2,8 / 21 ; 38 \%$ ). 

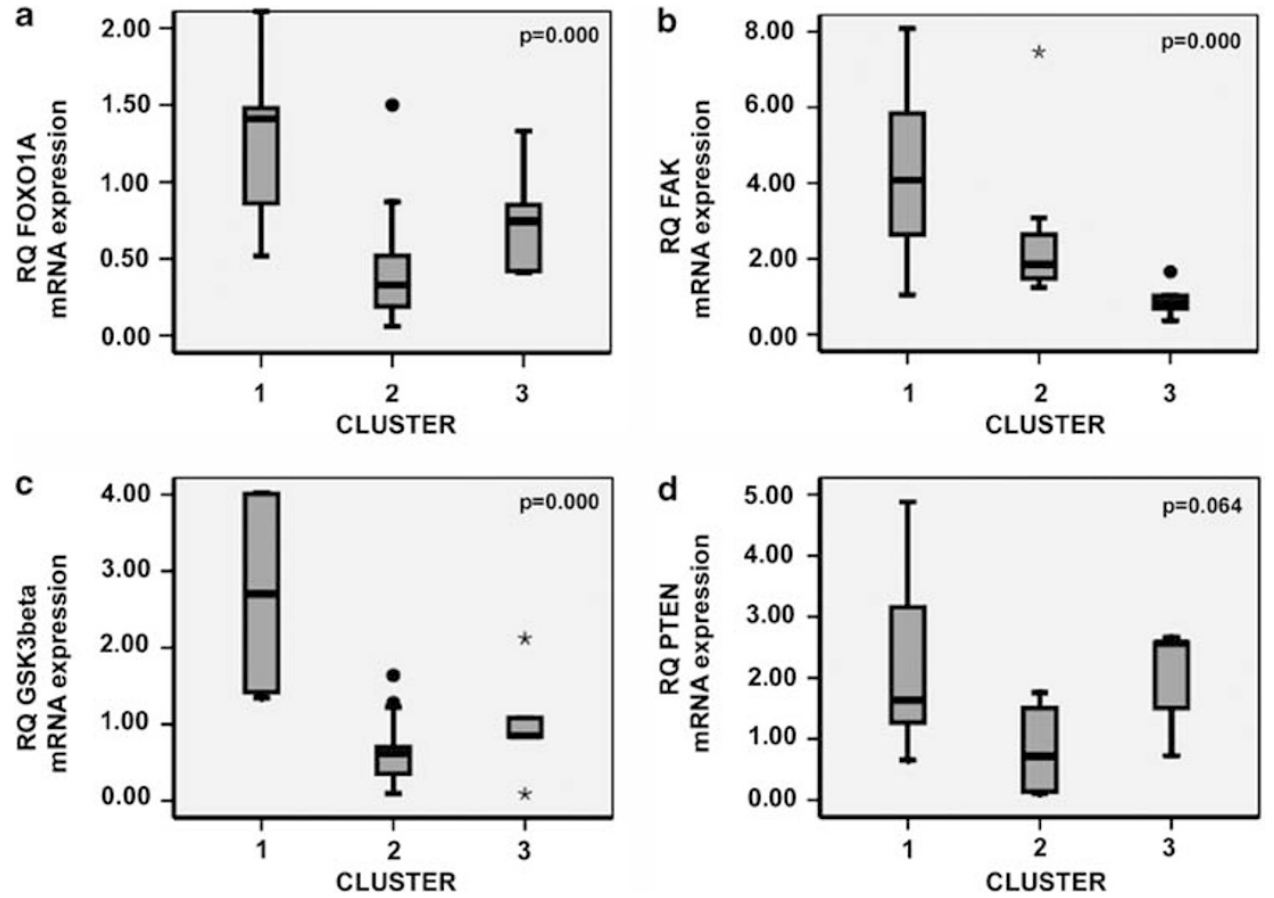

Figure 2 Plot box graphs representing RQ values expression for FOXO1A (a), FAK (b), GSK3- $\beta$ (c), and PTEN (d) in the three clusters.

The cases have been previously subjected to determination of the most frequent molecular genetic alterations of endometrial carcinomas, including microsatellite instability status, and mutational analysis of PTEN, PIK3CA, and p53 genes. ${ }^{16,25}$ Only PTEN mutations and p53 alterations varied significantly according to the tumor distribution into the three clusters $(P=0.017$ and $P=0.000$, respectively). PTEN mutations were more frequent in cluster 2 tumors $(10 / 17 ; 59 \%)$ than in cluster 1 carcinomas $(4 / 15 ; 27 \%)$. Moreover, no PTEN mutation was found in cluster 3. P53 alterations were present in all cluster 3 tumors $(6 / 6 ; 100 \%)$, in some cluster 1 tumors $(4 / 14 ; 29 \%)$, and in none of cluster 2 tumors. Carcinomas with exon 20 PIK3CA mutations (seven cases) were mostly high-grade carcinomas, and were almost all segregated into cluster 1 (five cases). None was found in cluster 3. In contrast, tumors that carried exon 9 PIK3CA mutations (four cases) were mostly low-grade endometrioid carcinomas (three cases), and were segregated into clusters 2 and 3 . None was encountered in cluster 1 . MI was found more commonly in cluster 2 $(6 / 17 ; 35 \%)$ than in cluster $1(3 / 15 ; 20 \%)$ or cluster 3 carcinomas $(0 / 6 ; 0 \%)$, but this association was not statistically significant. P16 protein was strongly expressed (score $>150)$ in 14 of $33(42 \%)$ endometrial tumors, more frequently in mixed endometrioid and non-endometrioid carcinomas $(4 / 4 ; 100 \%)$, and non-endometrioid carcinomas $(6 / 7 ; 86 \%)$ than in high-grade endometrioid carcinomas $(2 / 6 ; 33 \%)$ or in low-grade endometrioid carcinomas (2/16; $13 \% ; P=0.000$ ). Expression of p16 protein varied significantly according to the tumor distribution into the three clusters $(P=0.000)$; that is, it was strongly positive in all cluster 3 tumors (5/5), $62 \%$ $(8 / 13)$ cluster 1 tumors, and only $7 \%(1 / 15)$ of cluster 2 tumors.

\section{Discussion}

The PI3K-AKT pathway is frequently deregulated in various human epithelial tumors, including endometrial carcinomas. Alterations include mutation or amplification of PIK3CA, loss of PTEN function, mutation or amplification of $A K T$, and activation of growth factor receptors. Deregulated PI3K-AKT signaling pathway has been associated with aggressive tumor phenotype and poor prognostic parameters, including high grade, large size, nodal involvement, vascular invasion, and high proliferative fraction in breast cancer. ${ }^{3-9}$

Microarrays and real-time reverse transcriptionPCR (qRT-PCR) analyses for identification of differences in gene expression patterns have increased our understanding of relevant genetic events, and have contributed to further characterize the molecular genetic model for carcinogenesis. Gene expression profiling coupled with statistical method by clustering analysis is an important tool to evaluate genetic heterogeneity in carcinomas and is useful to develop expression-based classifications for many types of cancer, as well as markers of disease outcome. ${ }^{29,30}$ 
Table 4 Clinical and molecular features of 38 endometrial carcinomas distributed in the three cluster subgroups

Total Cluster 1 Cluster 2 Cluster 3 Pn (\%) $15(39 \%) \quad 17(45 \%) \quad 6(16 \%)$ value $^{\mathrm{a}}$

\begin{tabular}{|c|c|c|c|c|c|}
\hline \multicolumn{6}{|l|}{ Histological subtype } \\
\hline EEC low grade & 17 & 1 & 15 & 1 & \multirow[t]{4}{*}{0.000} \\
\hline EEC high grade & 8 & 5 & 1 & 2 & \\
\hline NEEC & 8 & 7 & 0 & 1 & \\
\hline EEC-NEEC & 5 & 2 & 1 & 2 & \\
\hline \multicolumn{6}{|l|}{ Grade } \\
\hline 1 & 8 & 0 & 8 & 0 & \multirow[t]{3}{*}{0.000} \\
\hline 2 & 9 & 1 & 7 & 1 & \\
\hline 3 & 21 & 14 & 2 & 5 & \\
\hline \multicolumn{6}{|l|}{ Vascular invasion } \\
\hline No & 25 & 10 & 12 & 3 & \multirow{2}{*}{ NS } \\
\hline Yes & 13 & 5 & 5 & 3 & \\
\hline \multicolumn{6}{|l|}{ Stage } \\
\hline 1 & 28 & 9 & 15 & 4 & \multirow[t]{4}{*}{0.094} \\
\hline 2 & 2 & 2 & 0 & 0 & \\
\hline 3 & 5 & 3 & 2 & 0 & \\
\hline 4 & 3 & 1 & 0 & 2 & \\
\hline \multicolumn{6}{|l|}{ Myometrial invasion } \\
\hline$<50 \%$ & 21 & 10 & 7 & 4 & \multirow[t]{2}{*}{ NS } \\
\hline$>50 \%$ & 17 & 5 & 10 & 2 & \\
\hline \multicolumn{6}{|l|}{ PIK3CA mutations } \\
\hline No & 27 & 10 & 13 & 4 & \multirow[t]{2}{*}{ NS } \\
\hline Yes & 11 & 5 & 4 & 2 & \\
\hline \multicolumn{6}{|l|}{ PTEN mutations } \\
\hline No & 24 & 11 & 7 & 6 & \multirow[t]{2}{*}{0.017} \\
\hline Yes & 14 & 4 & 10 & 0 & \\
\hline \multicolumn{6}{|l|}{ Microsatellite status } \\
\hline MS & 29 & 12 & 11 & 6 & \multirow[t]{2}{*}{ NS } \\
\hline MI & 9 & 3 & 6 & 0 & \\
\hline \multicolumn{6}{|l|}{ P53 alterations } \\
\hline No & 25 & 10 & 15 & 0 & \multirow[b]{2}{*}{0.000} \\
\hline Yes & 10 & 4 & 0 & 6 & \\
\hline \multicolumn{6}{|c|}{ P16 immunoexpression } \\
\hline No (score $\leq 150$ ) & 19 & 5 & 14 & 0 & \multirow[t]{2}{*}{0.000} \\
\hline Yes $($ score $>150)$ & 14 & 8 & 1 & 5 & \\
\hline
\end{tabular}

EEC, endometrioid endometrial carcinoma; NEEC, non-endometrioid endometrial carcinoma; NS, nonsignificant.

${ }^{\mathrm{a}} P$-values were calculated using Fisher's test.

In this study, we have analyzed the gene expression profile differences of the PI3K-AKT signaling pathway in 38 endometrial carcinomas, including endometrioid, non-endometrioid, and mixed endometrioid and non-endometrioid carcinomas. Gene expression analyses were performed at mRNA level by TLDA, ${ }^{31-33}$ and the gene expression pattern was analyzed by a hierarchical clustering analysis. ${ }^{28}$

Two groups of high-grade endometrial carcinomas with different molecular alterations were identified. One group (cluster 1) had exon 20 PIK3CA and/or PTEN mutations and the other (cluster 3) had p53 alterations and exon 9 PIK3CA mutations. Exons 9 and 20 of PIK3CA encode different domains and it is reasonable to speculate that they may have different oncogenic potential. ${ }^{34-36}$ These findings are compatible with our recent study in which mutations of exons 9 and 20 had prognostic implications. ${ }^{16,25}$ Although both groups of tumors have similar clinicopathological features, our results support the existence of a dualistic model for high-grade endometrial carcinomas.

A recent study has reconfirmed that the PIK3CA mutations occur almost exclusively in invasive tumors, whereas upstream mutations of the PI3KAKT pathway (PTEN and K-RAS mutations) occur with equal frequency in early- and advanced-stage tumors. This finding suggests that the PIK3CA mutations cooperate with these alterations in the malignant transformation. ${ }^{37}$ In addition, an integrated analysis of genome-wide expression and copy-number data disclosed two major clusters of primary endometrial carcinomas with different phenotype and prognosis. Amplifications of 3q26.32, harboring the oncogene PIK3CA, were associated with poor prognosis and segregated with the aggressive transcriptional cluster. This finding emphasizes the involvement of the PI3K-AKT pathway in aggressive endometrial carcinomas. ${ }^{38}$

P16 protein overexpression has been recently proposed as a marker of high-grade endometrial carcinoma. In our series, we confirmed this finding as we found intense p16 overexpression in both clusters of high-grade endometrial carcinomas. Our results are similar to those obtained by a recent comparative analysis of 117 endometrial carcinomas; that is, strong immunoexpression of p16 was identified in $16 \%$ of endometrioid carcinomas $(7 \%$ of low-grade endometrioid carcinomas, and $25 \%$ of high-grade endometrioid carcinomas), and $77 \%$ of non-endometrioid carcinomas $(92 \%$ of serous carcinomas, and $45 \%$ of clear cell carcinomas). These findings suggest that p16 protein expression correlates with tumor type and degree of differentiation, and it is useful for the differential diagnosis of endometrial carcinomas. $^{39}$ It has been confirmed that overexpression of p16 in serous endometrial carcinomas is unrelated to human papilloma virus infection..$^{40}$

Advances in the understanding of molecular genetic events in endometrial carcinomas has led to the development of novel and individualized therapeutic strategies. Although surgery is still the main treatment option, ${ }^{23,24}$ identification of two groups of high-grade endometrial carcinomas with different molecular alterations in the PIK3-AKT pathway can facilitate additional therapeutic strategies in these patients. Thus, further studies on the response to chemotherapy on patients with tumors in the two subgroups are justified. In fact, there are actually inhibitors of PI3K and mTOR that may be used as potential anticancer agents. ${ }^{41}$

In summary, hierarchical clustering analysis of genes involved in the PI3K-AKT pathway revealed two subgroups of high-grade endometrial carcinomas 
that correlated with the presence or absence of p53 alterations and the activation of the PI3K-AKT pathway (PTEN and/or exon 20 PIK3CA mutations). PI3K-AKT pathway alterations and p53 abnormalities may have distinct roles in high-grade endometrial tumorigenesis. Identification of these subgroups can provide insight into the biology of these tumors and may facilitate the development of future treatments.

\section{Acknowledgements}

This work was supported by Grants FIS PI04-1891, PI06-0950, PI07-0899, PI08-0410, and RTICCFIS RD06/0020/0015, Department of Health, Spain, Marató TV3 050432, and Fundacion Mutua Madrileña-07. We thank Dr A Gallardo for his assistance in evaluating the immunohistochemical stains and performing statistical analysis.

\section{Disclosure/conflict of interest}

The authors declare no conflict of interest.

\section{References}

1 Vivanco I, Sawyers CL. The phosphatidylinositol 3-kinase AKT pathway in human cancer. Nat Rev Cancer 2002;2:489-501.

2 Cully M, You H, Levine AJ, et al. Beyond PTEN mutations: the PI3K pathway as an integrator of multiple inputs during tumorigenesis. Nat Rev Cancer 2006;6:184-192.

3 Chakravarti A, Zhai G, Suzuki Y, et al. The prognostic significance of phosphatidylinositol 3-kinase pathway activation in human gliomas. J Clin Oncol 2004;22:1926-1933.

4 Tang JM, He QY, Guo RX, et al. Phosphorylated Akt overexpression and loss of PTEN expression in nonsmall cell lung cancer confers poor prognosis. Lung Cancer 2006;51:181-191.

5 Saal LH, Johansson P, Holm K, et al. Poor prognosis in carcinoma is associated with a gene expression signature of aberrant PTEN tumor suppressor pathway activity. Proc Natl Acad Sci USA 2007;104: 7564-7569.

6 Opel D, Poremba C, Simon T, et al. Activation of Akt predicts poor outcome in neuroblastoma. Cancer Res 2007;67:735-745.

7 Kato S, Iida S, Higuchi T, et al. PIK3CA mutation is predictive of poor survival in patients with colorectal cancer. Int J Cancer 2007;121:1771-1778.

8 Ogino S, Nosho K, Kirkner GJ, et al. PIK3CA mutation is associated with poor prognosis among patients with curatively resected colon cancer. J Clin Oncol 2009;27:1477-1484.

9 Aleskandarany MA, Rakha EA, Ahmed MA, et al. PIK3CA expression in invasive breast cancer: a biomarker of poor prognosis. Breast Cancer Res Treat; published online 22 August 2009; e-pub ahead of print.

10 American Cancer Society. Cancer Facts and Figures 2009. American Cancer Society: Atlanta, GA, 2009.
11 Bokhman JV. Two pathogenetic types of endometrial carcinoma. Gynecol Oncol 1983;15:10-17.

12 Prat J, Gallardo A, Cuatrecasas M, et al. Endometrial carcinoma: pathology and genetics. Pathology 2007; 39:72-87.

13 Lax SF. Molecular genetic pathways in various types of endometrial carcinoma: from a phenotypical to a molecular-based classification. Virchows Arch 2004; 444:213-223.

14 Catasus L, Machin P, Matias-Guiu X, et al. Microsatellite instability in endometrial carcinomas clinicopathologic correlations in a series of 42 cases. Hum Pathol 1998;29:1160-1164.

15 Bussaglia E, del Rio E, Matias-Guiu X, et al. PTEN mutations in endometrial carcinomas: a molecular and clinicopathologic analysis of 38 cases. Hum Pathol 2000;31:312-317.

16 Catasus L, Gallardo A, Cuatrecasas M, et al. PIK3CA mutations in the kinase domain (exon 20) of uterine endometrial adenocarcinomas are associated with adverse prognostic parameters. Mod Pathol 2008;21: 131-139.

17 Machin P, Catasus L, Pons C, et al. CTNNB1 mutations and beta-catenin expression in endometrial carcinomas. Hum Pathol 2002;33:206-212.

18 Moreno-Bueno G, Hardisson D, Sánchez C, et al. Abnormalities of the APC/beta-catenin pathway in endometrial cancer. Oncogene 2002;21:7981-7990.

19 Lagarda H, Catasus L, Arguelles R, et al. K-ras mutations in endometrial carcinomas with microsatellite instability. J Pathol 2001;193:193-199.

20 Strang P, Nordstöm B, Nilsson S, et al. Mutant p53 protein as a predictor of survival in endometrial carcinoma. Eur J Cancer 1996;32A:598-602.

21 Lax SF, Kendall B, Tashiro H, et al. The frequency of p53, K-Ras mutations, and microsatellite instability differs in uterine endometrioid and serous carcinoma: evidence of distinct molecular genetic pathways. Cancer 2000;88:814-824.

22 Sherman ME, Bur ME, Kurman RJ. P53 in endometrial cancer and its putative precursors: evidence for diverse pathways of tumorigenesis. Hum Pathol 1995;26:1268-1274.

23 Markman M. Unresolved issues in the chemotherapeutic management of gynecologic malignancies. Semin Oncol 2006;33:S33-S38.

24 Boruta II DM, Gehrig PA, Fader AN, et al. Management of women with uterine papillary serous cancer: a Society of Gynecologic Oncology (SGO) review. Gynecol Oncol 2009;115:142-153.

25 Catasus L, Gallardo A, Cuatrecasas M, et al. Concomitant PI3K-AKT and p53 alterations in endometrial carcinomas are associated with poor prognosis. Mod Pathol 2009;22:522-529.

26 Bader AG, Kang S, Zhao L, et al. Oncogenic PI3K deregulates transcription and translation. Nat Rev Cancer 2005;5:921-929.

27 Samuels Y, Ericson K. Oncogenic PI3K and its role in cancer. Curr Opin Oncol 2006;18:77-82.

28 Eisen MB, Spellman PT, Brown PO, et al. Cluster analysis and display of genome-wide expression patterns. Proc Natl Acad Sci USA 1998;95: 14863-14868.

29 Ge X, Yamamoto S, Tsutsumi S, et al. Interpreting expression profiles of cancers by genome-wide survey of breadth of expression in normal tissues. Genomics 2005;86:127-141. 
30 van't Veer LJ, Dai H, van de Vijver MJ, et al. Gene expression profiling predicts clinical outcome of breast cancer. Nature 2002;415:530-536.

31 Bustin S. Quantification of mRNA using real-time reverse transcription PCR (RT-PCR): trends and problems. J Mol Endocrinol 2002;29:23-39.

32 Akilesh S, Shaffer DJ, Roopenian D. Customized molecular phenotyping by quantitative gene expression and pattern recognition analysis. Genome Res 2003;13:1719-1727.

33 Abruzzo L, Lee K, Fuller A, et al. Validation of oligonucleotide microarray data using microfluidic lowdensity arrays: a new statistical method to normalize real-time RT-PCR data. BioTechniques 2005;38:785-792.

34 Gymnopoulos M, Elsliger M-A, Vogt PK. Rare cancer specific mutations in PIK3CA show gain of function. Proc Natl Acad Sci USA 2007;104:5569-5574.

35 Huang CH, Mandelker D, Schmidt-Kittler O, et al. The structure of a human p110alpha/p85alpha complex elucidates the effects of oncogenic PI3Kalpha mutations. Science 2007;318:1744-1748.

36 Zhao L, Vogt PK. Helical domain and kinase domain mutations in p110alfa of phosphatidylinositol 3-kinase induce gain of function by different mechanisms. Proc Natl Acad Sci USA 2008;105:2652-2657.

37 Oda K, Okada J, Timmerman L, et al. PIK3CA cooperates with other phosphatidylinositol $3^{\prime}$-kinase pathway mutations to effect oncogenic transformation. Cancer Res 2008:68:8127-8136.

38 Salvesen HB, Carter SL, Mannelqvist M, et al. Integrated genomic profiling of endometrial carcinoma associates aggressive tumors with indicators of PI3 kinase activation. Proc Natl Acad Sci USA 2009;106: 4834-4839.

39 Reid-Nicholson M, Iyengar $\mathrm{P}$, Hummer AJ, et al. Immunophenotypic diversity of endometrial adenocarcinomas: implications for differential diagnosis. Mod Pathol 2006;19:1091-1100.

40 Chiesa-Vottero AG, Malpica A, Deavers MT, et al. Immunohistochemical overexpression of p16 and p53 in uterine serous carcinoma and ovarian high-grade serous carcinoma. Int J Gynecol Pathol 2007;26: 328-333.

41 Chon HS, Hu W, Kavanagh JJ. Targeted therapies in gynecologic cancers. Curr Cancer Drug Targets 2006;6: 333-363. 